\title{
Recomendaciones en enfermedad hepática crónica y trasplante hepático durante COVID-19 en pediatría
}

\author{
Recommendations for pediatric chronic liver disease and liver transplant \\ during COVID-19
}

\author{
Marcela Godoy ${ }^{\mathrm{a}}$, Natalia Zuloaga ${ }^{\mathrm{b}}$, Elizabeth Navarro ${ }^{\mathrm{c}}$, Humberto Soriano ${ }^{\mathrm{d}}$, Cecilia Vizcaya ${ }^{\mathrm{e}}$, \\ Lorena Rodríguez G. ${ }^{\mathrm{f}}$, Juan Ignacio Juanet ${ }^{\mathrm{g}}$, Rossana Faundez ${ }^{\mathrm{h}}$, Juan Cristóbal Gana ${ }^{\mathrm{d}}$
}

\author{
aUnidad de Gastroenterología Infantil, Servicio de Pediatría, Hospital Clínico San Borja Arriarán \\ bServicio de Pediatría. Hospital El Pino. Unidad de Gastroenterología Infantil. Dr. Exequiel González Cortés \\ unidad de Gastroenterología Infantil, Hospital Exequiel González Cortés. Departamento de Pediatría y Cirugía Infantil Campus Sur, Facultad de \\ Medicina, Universidad de Chile \\ ¿Departamento de Gastroenterología y Nutrición Pediátrica, División de Pediatría, Escuela de Medicina, Pontificia Universidad Católica de Chile \\ eDepartamento de Infectología Pediátrica, División de Pediatría, Escuela de Medicina, Pontificia Universidad Católica de Chile \\ fUnidad de Gastroenterología Infantil, Servicio de Pediatría Hospital San Juan de Dios. Unidad de Gastroenterología Infantil, Servicio de Pediatría, \\ Clínica Alemana de Santiago. Facultad de Medicina Clínica Alemana-Universidad del Desarrollo \\ gUnidad de Gastroenterología. Hospital de niños Dr. Roberto del Río. Clínica Las Condes \\ hUnidad de Gastroenterología Infantil. Servicio de pediatría. Hospital Clínico San Juan de Dios, Facultad de Medicina Universidad de Chile
}

Recibido: 3 de mayo de 2020; Aceptado para publicación: 21 de mayo de 2020

\begin{abstract}
Resumen
El brote del virus SARS-CoV-2 que comenzó a fines del año 2019 en China, se ha expandido a Chile y al mundo rápidamente. Hasta la fecha, en Chile, ha afectado a 18.435 personas con una letalidad en adultos de 1,4\%. Los pacientes pediátricos con enfermedades hepáticas crónicas son también susceptibles a COVID-19 y podrían tener una peor evolución. El objetivo es entregar recomendaciones sobre el tratamiento médico de pacientes pediátricos con daño hepático crónico (DHC), hepatitis autoinmune (HAI), Enfermedad de hígado graso no alcohólico (EHGNA) y trasplantados hepáticos (TH) en relación a COVID-19. Lo primordial es evitar el contagio y para esto, lo más importante es el lavado de manos, uso de mascarilla en espacios públicos y cerrados, como el distanciamiento social y evitar contacto con personas sintomáticas. Los pacientes con DHC, HAI, EHGNA y TH deben evitar los controles presenciales y favorecer la telemedicina. No existe evidencia que recomiende la modificación del tratamiento basal en estos casos. En pacientes COVID-19 (+) se recomienda medidas de aislamiento, preferir uso de paracetamol como antipirético y analgésico y en el manejo de la inmunosupresión, debe considerarse cada caso de forma individual, según gravedad y con evaluación del especialista. Además, se revisan las actuales terapias específicas para COVID-19 y sus precauciones en pacientes con hepatopatías. Las medidas de prevención del contagio, aislamiento social y diagnóstico precoz son fundamentales en pacientes con enfermedad hepática y el riesgo de infección por SARS$\mathrm{CoV}-2$.
\end{abstract}

Palabras clave: Coronavirus; Trasplante Hepático; Daño Hepático Crónico; Hepatitis Autoinmune 


\section{Abstract}

The SARS-CoV-2 virus outbreak, which began in late 2019 in China, has spread very quickly to Chile and worldwide. In Chile, we currently have around 18,435 people infected with $1.4 \%$ of adult mortality. Pediatric patients with chronic liver diseases (CLD) are susceptible as well to COVID-19 and could have a worse prognosis. The objective is to give recommendations about medical treatment to pediatric patients with chronic liver disease (CLD), autoimmune hepatitis (AIH), Non- Alcoholic fatty liver disease (NAFLD), and liver transplant in the context of COVID-19. The most important issue in the management of these patients is to avoid exposure to the virus, hand washing, the use of face masks in public and closed places, as well as social distancing, and avoiding contact with positive COVID-19 patients. In Children with CLD, AIH, NAFLD, and liver transplant, outpatient follow-up should be avoided when possible and replaced with videoconference consultation. No evidence recommends modifications to their baseline treatment. Positive COVID-19 patients should be isolated, the use of paracetamol as an antipyretic and analgesic and modifications to immunosuppressant drugs should be seen by the specialist in a case to case basis according to its severity. In addition, we reviewed current specific therapies for COVID-19 and their precautions in patients with liver disease. Protective measures, social distancing, and early diagnosis are very important in patients with liver disease to decrease the risk of SARS-CoV-2 infection.

\section{Keywords:}

Coronavirus; Liver

Transplant; Chronic

Liver Disease;

Autoimmune Hepatitis

\section{Introducción}

Luego de conocerse el brote del virus SARS-CoV-2 en China a fines de 2019, este se ha extendido por todo el mundo $\mathrm{o}^{1-4} \mathrm{y}$ ha llegado a Chile en pocos meses, afectando a la fecha de esta publicación a 18.435 pacientes en nuestro país de los cuales 247 (1,4\%) adultos han fallecido ${ }^{5}$. A la fecha no existen publicaciones pediátricas basadas en evidencia acerca de pacientes con patologías hepáticas crónicas en contexto de la pandemia por SARS-CoV2 ${ }^{6}$. La experiencia internacional sugiere que los pacientes inmunocomprometidos no presentan mayor riesgo de infección que la población general ${ }^{7,8}$. Aun así, una infección pulmonar por SARS-CoV2 podría tener una peor evolución en estos pacientes con daño hepático crónico o trasplantados, si extrapolamos a lo que ocurre con otros virus que causan mayor gravedad pulmonar en pacientes inmunodeficientes, como, por ejemplo, influenza, adenovirus, y virus respiratorio sincicial ${ }^{7}$. Si bien el COVID-19 produce principalmente compromiso respiratorio, se han descrito aumento de transaminasas y alteración de la función hepática en el curso de infecciones severas ${ }^{7,9}$.

Este documento busca entregar recomendaciones generales sobre el tratamiento médico de pacientes pediátricos con daño hepático crónico (DHC) y/o trasplantados hepáticos. Es importante mencionar, que estas recomendaciones son en base a la información disponible a la fecha, pero es necesario estar en continuo proceso de revisión de la literatura dado que es una enfermedad que está en continuo desarrollo y la información actual es limitada.

El diagnóstico en este grupo de pacientes debe rea- lizarse mediante PCR COVID-19 debido a que su respuesta serológica es impredecible ${ }^{10}$.

\section{Pacientes SIN COVID-19}

\section{Prevención general, lo más importante ${ }^{2,10}$ :}

- Enseñar al niño a lavar sus manos.

- Lavado de manos mínimo 20-30 segundos con agua y jabón.

- Higiene de manos con alcohol gel, duración 20 segundos.

- No tocar la cara con las manos.

- Estornudar o toser sobre el pliegue del codo o en pañuelo desechable, eliminar e higienizar las manos.

- Quedarse en casa. Si es indispensable salir de casa, mantener distancia de ojalá 2 metros con las otras personas y usar mascarilla.

- Evitar contacto con personas con síntomas respiratorios y/o gastrointestinales.

- Evitar contacto con personas enfermas confirmadas o contactos de pacientes con COVID-19.

\section{Recomendaciones para pacientes con DHC SIN COVID-19}

a) Debe evitarse el control presencial, a menos que tengan alguna descompensación aguda. Favorecer los controles no presenciales, tipo telemedici$\mathrm{na}^{2,8,10,11}$.

b) Se recomienda no modificar su tratamiento habitual de mantención.

c) Deben ser vacunados contra la influenza (idealmente también a familiares directos) y neumoco$\mathrm{CO}^{8}$. 
d) No se recomienda screening de várices esofágicas con endoscopía digestiva alta, sólo debe realizarse en caso de hemorragia aguda. Tampoco se recomienda hacer profilaxis endoscópica en pacientes estables. Estos procedimientos son de mayor riesgo de contagio y en caso de necesitar realizarlo, deben tomarse todas las medidas de precaución.

\section{Hepatitis autoinmune}

En el caso de pacientes con hepatitis autoinmune (HAI) recibiendo terapia inmunosupresora, existen protocolos de tratamiento de diferentes centros de referencia europeos implementados durante la pandemia. La reducción empírica de inmunosupresores no se recomienda en todos los casos, es necesario balancear el riesgo v/s beneficio de reducir y/o suspender el tratamiento inmunosupresor ya que, una reactivación de la HAI, requerirá mayor dosis de corticoides, lo cual aumenta el riesgo de infección grave ${ }^{12}$.

Según el riesgo de complicaciones se pueden dividir en pacientes ${ }^{13}$ :

Bajo riesgo: Pacientes con $\mathrm{HAI}$ estable, en tratamiento crónico.

- Evitar visitas a centro hospitalario, favorecer el uso de telemedicina.

- No realizar cambios en el tratamiento inmunosupresor de base.

- Programar entrega de fármacos de mantención para favorecer su uso continuo.

- En Centros hospitalarios se debe asegurar flujos diferenciados entre pacientes sintomáticos y asintomáticos, para que estos últimos puedan acudir con seguridad en caso necesario.

- Diferir, en lo posible, exámenes de laboratorio e imagenológicos. En aquellos casos que es necesario evaluar, realizar bajo estrictas medidas de protección y revisar vía telemedicina ${ }^{2,10}$.

- Educar en medidas de prevención general descritas anteriormente.

Alto riesgo: Pacientes con HAI y cirrosis descompensada que presenten alguna complicación asociada (hemorragia digestiva, ascitis, encefalopatía hepática, peritonitis bacteriana espontánea) o reagudización de HAI (ictericia colestásica en colangitis esclerosante primaria (CEP), colangitis aguda) o debut de HAI como insuficiencia hepática aguda. Presentan una alta morbimortalidad, independiente del cuadro viral asociado.

- Se requiere una evaluación de urgencia.

- En el acceso a urgencia se debe evitar contacto con pacientes COVID-19 (+), mediante flujos separados.

- Hospitalizar en unidades de aislamiento y realizar RPC COVID-19 (SARS-CoV-2).
- En reactivaciones de HAI y debut como falla hepática aguda, iniciar tratamiento estándar a dosis habituales.

- En caso de infecciones asociadas se debe evaluar la reducción o suspensión transitoria de la dosis de inmunosupresores, especialmente de metotrexato y azatioprina en pacientes que se encuentren con linfopenia.

- Realizar endoscopía digestiva alta sólo en pacientes con hemorragia aguda ${ }^{14}$.

- Intentar una estadía hospitalaria lo más corta posible.

\section{Enfermedad de hígado graso No alcohólico (EHGNA)}

Los datos emergentes sugieren que los pacientes adultos con EHGNA e IMC elevado pueden tener mayor riesgo de progresión a enfermedad severa por COVID-19 ${ }^{15}$. En los pocos casos severos reportados se ha evidenciado aumento leve de alanina aminotransferasa (ALT) o aspartato aminotransferasa (AST) $(<2 \mathrm{VN})$. Varios estudios además han demostrado un efecto de miositis viral, con alteración de creatinfosfoquinasa (CPK), lactato deshidrogenasa (LDH) o mioglobina, pudiendo contribuir a las alteraciones de AST, semejante a como lo hace la influenza. Considerando lo anterior y lo que sabemos actualmente de EHGNA en niños y adolescentes y el alto porcentaje asociado a obesidad, creemos que este grupo constituye una población de riesgo en caso de cursar con infección por COVID-19. Se recomienda:

a) Evitar control presencial. En caso de ser necesario exámenes de laboratorio de control, éstos pueden ser chequeados por telemedicina. Imágenes (ej. ecografía abdominal) solicitadas previamente, se pueden diferir.

b) Incentivar el autocuidado con respecto a:

- Mantener una dieta saludable y balanceada. Evitar bebidas azucaradas y alimentos ricos en fructosa.

- Estimular actividad física en domicilio: realizar ejercicio de intensidad moderada a alta.

- Regular tiempo de exposición a pantallas/internet: considerando cuarentena/clases de colegio en línea. Según recomiendan los expertos, hay que llegar a acuerdos sobre los tiempos que se destinaran para estar conectados.

\section{Recomendaciones para pacientes con trasplante hepático SIN COVID-19}

Hasta la fecha, hay 3 casos reportados de pacientes pediátricos trasplantados hepáticos con infección por SARS-CoV-2 $2^{1}$. Además, no hay evidencia actual de rol de la inmunosupresión como factor agravante, sin embargo, deben ser considerados como grupo de riesgo, hasta tener mayor evidencia. Deben man- 
tenerse las mismas recomendaciones de los pacientes de daño hepático crónico en cuanto a permanecer en domicilio ${ }^{10}$.

Es importante señalar que, dada la pandemia actual, se debe omitir los trasplantes hepáticos que no sean urgentes. En caso de trasplante que no pueda ser diferido, se debe realizar RPC COVID-19 en donante y receptor. Ambos resultados deben ser negativos para poder llevar a cabo el trasplante, ya que el riesgo del receptor es muy elevado al asociar la infección a inmunosupresión intensa ${ }^{2,8,16}$.

\section{Recomendaciones en DHC CON COVID-19 (+)}

\section{Tratamiento ambulatorio}

a) Medidas generales, incluidas aislamiento ${ }^{2,10}$

- El caso debe dormir en una pieza solo. Si no se puede, que tenga al menos un metro de distancia con otros y uso de mascarilla. La habitación debe tener ventilación.

- Moverse lo menos posible por la casa.

- Tener sus propios utensilios para comer, preferir toallas de papel y usar una toalla individual.

- Todos quienes viven con el paciente deben ser monitoreados por la autoridad sanitaria.

b) Preferir el uso de paracetamol, $15 \mathrm{mg} / \mathrm{kg} /$ dosis, sobre antiinflamatorios no esteroidales (AINES). El paracetamol también se puede usar por vía e.v. ${ }^{8}$.

c) Inmunosupresión: Estos tratamientos siempre deben considerar al especialista para la toma de decisiones individuales. Pacientes con inmuosupresores que se presenten con diarrea, deben consultar a sus tratantes ya que puede afectar los niveles y como consecuencia el curso de la enfermedad ${ }^{10}$.

- HAI: En los pacientes con enfermedad moderada a grave, se puede considerar la disminución o suspensión transitoria de azatioprina y micofenolato, manteniendo tratamiento con prednisona que debe considerarse dosis por lo menos que cubra el stress inflamatorio y evitar insuficiencia supra-renal.

- Trasplante hepático: En pacientes con curso moderado a grave de infección, se puede considerar la suspensión de azatioprina, en especial si hay sobreinfección o linfopenia. Para el tratamiento con Inhibidores de la calcineurina, se puede considerar la disminución de tratamiento, pero no la suspensión. En caso de estar en tratamiento con prednsiona, no se recomienda la suspensión y ajustar además por estado de stress inflamatorio y evitar insuficiencia suprarenal ${ }^{10}$. d) EHGNA con comorbilidad asociada (por ej. DMobesidad): realizar estudio con hemograma, perfil bioquímico (con LDH), perfil hepático, ferritina y CPK. En caso de elevación de transaminasas, considerar estudio del resto de etiologías probables además de SARS-CoV-2 (incluyendo estudio de virus hepatótropos). Es muy relevante evaluar la posibilidad de un síndrome inflamatorio descrito en pacientes con COVID-19, monitorizando leucopenia, trombocitopenia, ferritina, Dímero D, LDH e IL6 ${ }^{17}$.

e) Tratamientos específicos: hasta la fecha, no hay reportes comprobados de eficacia de tratamientos específicos en pacientes pediátricos con hepatopatías, por lo que debe evaluarse con especial cuidado, el balance entre posibles beneficios y por otro lado, hepatopatías o interacción con su tratamiento inmunosupresor. Se puede revisar información actualizada en https://www.covid19-druginteractions.org $/{ }^{18}$.

\section{Hospitalización en DHC y trasplante hepático}

a) Hospitalizar para observación por al menos $48 \mathrm{~h}$ para monitorización cercana y diagnosticar eventuales complicaciones.

b) En caso de presentarse con síntomas digestivos, los niveles de inmunupresión deben controlarse y ajustarse en cada caso, ya que pueden alterarse e influir en la evolución de COVID-19.

c) Es importante tener en consideración que los agentes terapéuticos utilizados en el manejo de los pacientes COVID-19 pueden ser hepatotóxicos. Si bien es poco común que la cloroquina, hidroxicloroquina, y azitromicina, causen elevación de transaminasas, las últimas recomendaciones sugieren no utilizar cloroquina e indicar hidroxicloroquina debe ser evaluado en forma supervisada y discutida en forma multidisciplinaria y nunca asociar con Azitromicina, por alto riesgo de prolongar QT y favorecer arritmias.

La presencia de alteración de transaminasas no debiera contraindicar su uso, sin embargo, con elevaciones $>5 \mathrm{VN}$ mejor no utilizar ${ }^{5}$. En caso de que las pruebas de función hepática empeoren, considerar evaluar otras causas como miositis (AST > ALT), daño hepático inducido por fármacos, isquemia, entre otras ${ }^{2}$.

d) Terapias específicas

1. Hidroxicloroquina: Existen reportes de complicaciones cardiológicas frecuentes en pacientes con esta terapia. Por este motivo, su uso debe ser con 
monitorización estricta y en pacientes hospitalizados. Se recomienda conversar con equipo de infectología la dosis al inicio y durante la evolución del tratamiento.

- Efectos adversos: Prolongación del intervalo QT y arritmias secundarias. Se recomienda tomar un electrocardiograma (ECG) antes del inicio de la terapia con hidroxicloroquina. Se sugiere evaluar uso de otros medicamentos que prolonguen el QT (quinolonas, macrólidos, voriconazol, ondansetron, metadona, clorpromazina, cisaprida, risperidona, domperidona). Tomar un segundo ECG de control a las $48 \mathrm{~h}$ de iniciado el tratamiento. Otros efectos adversos: hipoglicemia y toxicidad hematológica. Se sugiere evaluar glicemia y hemograma diariamente $^{19}$.

2. Tocilizumab: Como alternativa terapéutica de rescate en pacientes graves, con desregulación de la respuesta inflamatoria o con sospecha de síndrome hemofagocítico, se sugiere el uso de este anticuerpo monoclonal anti-IL-6 ${ }^{18}$.

- Efectos adversos: mayor riesgo de sobreinfecciones bacterianas.

- No utilizar en cirrosis descompensada. Puede reactivar virus hepatitis B. Monitorizar transaminasas (descrito elevación de enzimas hepáticas).

3. Lopinavir/ritonavir: Se usó este medicamento en pacientes con COVID-19, pero estudios más recientes no encuentran diferencias entre pacientes tratados y no tratados, por lo que no recomendamos su uso. Además, la administración de este medicamento tiene importantes interacciones con inmunosupresores utilizados en trasplantados hepáticos (inhibidores de calcineurina y de mTOR) ${ }^{18}$.

4. Remdesivir: Antiviral no disponible en Chile. Estudios muestran disminución de carga viral, pero aún no es claro el beneficio en los pacientes. Existe experiencia en pacientes con cirrosis, pero se estima que podría ser seguro debido a uso de otros antivirales de la misma clase en hepatitis B y C. Monitorizar transaminasas (descrito elevación de enzimas hepáticas $)^{18,20}$.
5. Plasma convaleciente: No existen estudios con uso de plasma convaleciente en niños con COVID-19, aunque existen reportes de su uso en niños con cuadros graves. Actualmente se están llevando a cabo estudios evaluando su uso en pacientes adultos con alto riesgo de una evolución desfavorable y uso en pacientes con cuadro grave en nuestro país y en varios países reportados en Clinicaltrials.gov. Debemos seguir esos protocolos para ver si el plasma inmune obtenido de pacientes recuperados de COVID-19, pudiera constituir una terapia útil en pacientes pediátricos inmunocomprometidos.

En casos de COVID-19 muy grave, se debería considerar la retirada total de la inmunosupresión ${ }^{21}$ tras evaluar el riesgo de rechazo y de pérdida del injerto no vital. Evaluar asociar IGIV policlonal por su efecto inmunomodulador ${ }^{18}$.

\section{Conclusiones}

Las medidas de prevención del contagio, aislamiento social y diagnóstico precoz son importantes en los pacientes pediátricos con enfermedad hepática y riesgo de infección por COVID-19. Esta infección se debe sospechar en pacientes con fiebre sin causa evidente y/o en pacientes son síntomas respiratorios o digestivos. En general se recomienda la mantención del tratamiento habitual en la mayoría de las patologías. Los controles presenciales se deben reducir lo más posible. La reducción empírica de inmunosupresores no se recomienda en todos los casos, es necesario balancear el riesgo vs beneficio de reducir y/o suspender el tratamiento inmunosupresor, que siempre debe ser consultado con el especialista.

\section{Conflicto de intereses}

Los autores declaran no tener conflicto de intereses.

\section{Referencias}

1. Matthai J, Shanmugam N, Sobhan P. Coronavirus Disease (COVID-19) and the Gastrointestinal System in Children. Indian Pediatr 2020 Apr 12. pii: S097475591600162. [Epub ahead of print].

2. AASLD. Clinical Insights for Hepatology and Liver Transplant providers during the COVID-19 Pandemic. 23 de marzo de 2020. https://www.aasld.org/sites/ default/files/2020-03/AASLD-COVID19ClinicalInsights-3.23.2020-FINAL-v2.pdf

3. Guan W, Ni Z, Hu Y, et al. Clinical

characteristics of coronavirus

disease 2019 in China. N Engl J Med 2020

Feb 28. doi: 10.1056/NEJMoa2002032.
[Epub ahead of print].

4. Yang X, Yu Y, Xu J, et al. Clinical course and outcomes of critically ill patients with SARS-CoV-2 pneumonia in Wuhan, China: a single-centered, retrospective, observational study. Lancet Respir Med. 2020 Feb 24 [epub ahead of print]. doi: 10.1016/S2213-2600(20)30079-5.

5. Fuente Minsal. 
6. Zhu N, Zhang D, Wang W, et al. A Novel Coronavirus from Patients with Pneumonia in China, 2019. N Engl J Med. 2020;382(8):727-33. doi: 10.1056/ NEJMoa2001017. Epub 2020 Jan 24

7. D'Antiga L. Coronaviruses and immunosuppressed patients. The facts during the third epidemic. Liver Transpl 2020 Mar. doi: 10.1002/lt.25756. [Epub ahead of print]

8. Boettler T, Newsome PN, Mondelli MU, et al. Care of patients with liver disease during the COVID-19 pandemic: EASLESCMID position paper. JHEP Reports (2020). doi: https://doi.org/10.1016/j. jhepr.2020.100113.

9. Zhang C, Shi L, Wang FS. Liver injury in COVID-19: management and challenges. Lancet Gastroenterol Hepatol. 2020;5(5):428-430. doi: 10.1016/ S2468-1253(20)30057-1. Epub 2020 Mar 4.

10. Liu H, He X, Wang Y, et al. Management of COVID-19 in patients after liver transplantation: Beijing working party for liver transplantation. Hepatol Int. 2020. Apr 10. doi: 10.1007/s12072-020-10043-Z. [Epub ahead of print].

11. Tapper EB, Asrani SK. COVID-19 pandemic will have a long-lasting impact on the quality of cirrhosis care. J Hepatol. 2020 Apr 13. pii: S0168-8278(20)30217-8. doi: 10.1016/j.jhep.2020.04.005. [Epub ahead of print].

12. Lleo A, Invernizzi P, Lohse AW, Aghemo A, Carbone M. Highlights for management of patients with Autoimmune Liver Disease during COVID-19 pandemia, J Hepatol. 2020 Apr 10. https://doi.org/10.1016/j. jhep.2020.04.002.

13. Huang $\mathrm{C}$, Wang $\mathrm{Y}, \mathrm{Li} \mathrm{X}$, et al. Clinical features of patients infected with 2019 novel coronavirus in Wuhan, China. Lancet 2020;395:497-506.

14. Repici A, Maselli R, Colombo M, et al. Coronavirus (COVID-19) outbreak: what the department of endoscopy should know. Gastrointest Endosc 2020.

15. Ji D, Qin E, Xu J, et al. G. Non alcoholic fatty liver diseases in patients with COVID-19: A retrospective study. J Hepatol. 2020 Apr 8. pii: S01688278(20)30206-3. doi: 10.1016/j. jhep.2020.03.044. [Epub ahead of print].

16. Qin J, Wang H, Qin X, et al. Perioperative Presentation of COVID-19 Disease in a Liver Transplant Recipient. Hepatology. 2020 Mar 27. doi: 10.1002/hep.31257. [Epub ahead of print]

17. Prins GH, Olinga P. Potential implications of COVID-19 in nonalcoholic fatty liver disease. Liver Int. 2020
Apr 19. doi: 10.1111/liv.14484. [Epub ahead of print].

18. Grupo de Trabajo de COVID-19 en el Trasplante de Órgano Sólido (Corona-TOS) Grupo de Estudio de Infección en el Trasplante y el Huésped Inmunocomprometido (GESITRA-IC) de la Sociedad Española de Enfermedades Infecciosas y Microbiología Clínica (SEIMC) Red Española de Investigación en Patología Infecciosa (REIPI). Recomendaciones para el tratamiento de receptores de TOS con diagnóstico de COVID-19. https://www.setrasplante.org/ noticiashome/65.

19. Hydroxychloroquine sulfate. https://www. accessdata.fda. gov/drugsatfda_docs/label /2017/009768s037s045s047lbl.pdf.

20. Chiotos K1, Hayes M, Kimberlin DW, Jones SB, James SH, Pinninti SG. Multicenter initial guidance on use of antivirals for children with COVID-19/ SARS-CoV-2. J Pediatric Infect Dis Soc. 2020 Apr 22. pii: piaa045. doi: 10.1093/ jpids/piaa045. [Epub ahead of print].

21. Bin L, Yangzhong W, Yuanyuan Z, Huibo S, Fanjun Z, Zhishui C. Successful Treatment of Severe COVID-19 Pneumonia in a Liver Transplant Recipient. Am J Transplant. 2020 Apr 3. doi: 10.1111/ajt.15901. [Epub ahead of print]. 